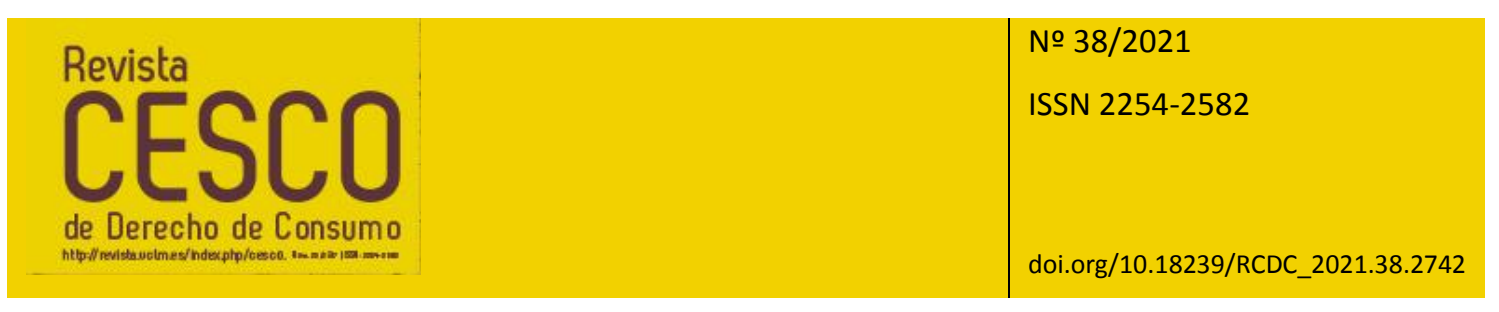

\title{
PROPUESTA DE REgLAMENTO EN MATERIA DE RESPONSABILIDAD CIVIL POR EL USO DE INTELIGENCIA ARTIFICIAL, DEL PARLAMENTO EUROPEO, DE 20 DE OCTUBRE DE 2020*
}

\author{
Pilar Álvarez Olalla \\ Catedrática de Derecho Civil \\ Universidad Rey Juan Carlos
}

Resumen: El Parlamento Europeo ha aprobado una Propuesta de Reglamento en materia de responsabilidad civil por el uso de sistemas de inteligencia artificial, publicada el 20 de octubre de 2020. En ella se establece una distinción entre los denominados Sistemas de Alto Riesgo, que estarán sometidos a un régimen de responsabilidad objetiva impuesto a los operadores de dicho sistema, si bien con límites indemnizatorios muy severos; y los Sistemas no de Alto Riesgo, en los cuales el operador queda sometido a un sistema de responsabilidad cuasiobjetiva, con inversión de la carga de la prueba de la culpa, pero sin límites indemnizatorios predeterminados.

Palabras clave: Inteligencia artificial, Responsabilidad civil, Responsabilidad objetiva, Responsabilidad cuasiobjetiva.

Title: Proposal for a regulation of the European Parliament and of the Council on liability for the operation of Artificial Intelligence, 20th October 2020

\footnotetext{
Abstract: European Parliament has adopted a Proposal for a Regulation on liability for the operation of artificial Intelligence-systems, published on 20th October 2020. This proposal treats high risk AI systems differently from other systems not considered so dangerous. So, High risk AI systems operators are subject to a strict liability regime, albeit with very severe compensation limits; while systems that are

* Trabajo realizado en el marco del Proyecto de Investigación RTI2018-097418-B-I00 del Ministerio de Ciencia, Innovación y Universidades (MCIU) y la Agencia Estatal de Investigación (AEI) cofinanciado por el Fondo Europeo de Desarrollo Regional (FEDER) titulado "Nuevas Perspectivas de la Responsabilidad Civil. Revisión de la Imputación Objetiva como criterio delimitador de la Causalidad" del que soy Investigadora Principal.
} 
not high risk, are subject to a fault-based liability system, with reversal of burden of proof, and without specific compensation limitis.

Keywords: Artificial Intelligence, Civil Liability, Strict Liability, fault-based liability, reversal of burden of proof.

SUMARIO: 1. Cuestiones previas. 2. Sujetos responsables. 3. El sistema de responsabilidad civil diseñado en la propuesta. 3.1. Sistemas de Alto Riesgo. Responsabilidad objetiva. 3.2. Otros Sistemas de IA (no de Alto Riesgo). Responsabilidad Subjetiva. 4. Disposiciones comunes aplicables a Sistemas de IA ya sean o no de Alto Riesgo. 4.1. Culpa Concurrente de la víctima. 4.2. Responsabilidad solidaria de los operadores y acciones de regreso.

\section{Cuestiones previas}

Con posterioridad a la Resolución de 2017, el Parlamento ha continuado su labor, elaborando informes y documentos que impulsen la regulación normativa de la inteligencia artificial. En junio de 2020 presentó el estudio "Artificial Intelligence: How does it work, why does it matter, and what can we do about it"1, si bien este trabajo no afectaba en gran medida a las cuestiones de responsabilidad civil. En septiembre de 2020 se crea la "Comisión especial para la inteligencia artificial" del Parlamento. El 20 de octubre de 2020 se aprueban tres documentos de especial relevancia. Una Resolución centrada en las cuestiones éticas "Framework of ethical aspects of artificial intelligence, robotics and related technologies" 2020/2012(INL) ${ }^{2}$, que incluye propuestas sobre cómo evitar sesgos y vulneración de los derechos fundamentales; una Resolución centrada en las normas de responsabilidad civil "Civil liability regime for artificial intelligence" 2020/2014(INL)) ${ }^{3}$, con el objetivo de proteger a los ciudadanos, a fin de generar confianza en el uso de las aplicaciones de IA. El tercer documento es un Informe centrado en los derechos de propiedad intelectual "Report on intelectual property rights for the development of artificial intelligence technologies" 2020/2015 (INI) ${ }^{4}$. Además, la Comisión de Asuntos Jurídicos trabaja en las implicaciones civiles y militares del Inteligencia artificial ${ }^{5}$; la Comisión de Cultura analiza el impacto de la IA en la educación, cultura y sector audiovisual ${ }^{6}$; y la Comisión de libertades civiles la incidencia del uso de la IA en el Derecho Penal?. En este caso, nos centraremos en el segundo de los documentos citados, la Resolución de 20 de octubre de 2020 en materia de responsabilidad civil.

Esta Resolución opta por un sistema de armonización plena mediante la aprobación de un Reglamento que proporcione una legislación uniforme para todos los Estados

\footnotetext{
${ }^{1}$ https://www.europarl.europa.eu/RegData/etudes/STUD/2020/641547/EPRS STU(2020)641547 EN.pdf

2 Se trata de una Propuesta de Informe del que ha sido ponente el eurodiputado español (PSOE) Ibán García del Blanco, y que pone al ser humano en el centro de la Propuesta. Incluye una Propuesta de regulación de principios éticos para el desarrollo, despliegue y uso de la inteligencia artificial. https://www.europarl.europa.eu/doceo/document/TA-9-2020-0275 EN.html

${ }^{3}$ https://www.europarl.europa.eu/doceo/document/TA-9-2020-0276 EN.html

4 https://www.europarl.europa.eu/doceo/document/A-9-2020-0176 EN.html

${ }^{5}$ https://oeil.secure.europarl.europa.eu/oeil/popups/ficheprocedure.do?reference $=2020 / 2013$ (INI) \&l=en

6 https://oeil.secure.europarl.europa.eu/oeil/popups/ficheprocedure.do?reference=2020/2017(INI)\&l=en

7 https://oeil.secure.europarl.europa.eu/oeil/popups/printficheglobal.pdf?id=710029\&l=en
} 
Revista CESCO, N. $37 / 2021$

doi.org/10.18239/RCDC_2021.38.2742
Pilar Álvarez Olalla

Propuesta de Reglamento en materia de responsabilidad civil por el uso de inteligencia artificial, del Parlamento Europeo, de 20 de octubre

de 2020

miembros, sin que ello suponga una modificación completa de los regímenes de responsabilidad tanto de la UE como de las legislaciones internas. Por ello, incluye una Propuesta de Reglamento relativo a la responsabilidad civil por el funcionamiento de los sistemas de inteligencia artificial. En su Preámbulo, la Propuesta recoge ideas ya incluidas en el Informe del Grupo de Expertos de la Comisión, de noviembre de $2019^{8}$, en el Informe de la Comisión ${ }^{9}$ y en el Libro Blanco ${ }^{10}$ sobre esta materia, de 19 de febrero de 2020, tales como la dificultad que entraña para la víctima la prueba de la causa del daño, dadas las características de opacidad, conectividad, y autonomía de los sistemas; o la necesidad de respeto al principio de equivalencia funcional, en virtud del cual una víctima de un daño causado por IA debe ser indemnizada de igual manera que si el daño se hubiera causado de cualquier otro modo. La Propuesta trata de dar una respuesta ponderada a la necesidad de indemnizar a las víctimas a fin de crear un sistema que dé confianza en el uso de la inteligencia artificial, en relación con la necesidad de no lastrar la innovación de la tecnología y dar seguridad jurídica a las empresas respecto a qué riesgos relacionados con posibles indemnizaciones por daños asumen.

\section{Sujetos responsables}

La Propuesta de Reglamento admite que uno de los sujetos que puede ser responsable del daño sea el productor, caso en el que considera preferible mantener su responsabilidad en el ámbito de la Directiva 85/347 de responsabilidad por daños causados por productos defectuosos, si bien se prevé que esta sufra las necesarias modificaciones. De hecho, se considera que ambos, tanto el Reglamento propuesto como la Directiva, debidamente modificada para adaptarse a las características de la IA, "son los dos pilares de un marco común de responsabilidad civil para los sistemas de IA" y plantea a la Comisión la posibilidad de reflexionar sobre la conveniencia de regular la responsabilidad por productos a través de un Reglamento, en lugar de a través de una Directiva. También sugiere la modificación del concepto de "producto", así como la del concepto "fabricante o productor". El primero, para incluir los contenidos y servicios digitales; $y$, el segundo, para incluir fabricantes, desarrolladores, programadores, prestadores de servicios y operadores iniciales ${ }^{11}$. También se sugiere la posibilidad de invertir la carga de la prueba del defecto para casos predefinidos, tras una exhaustiva evaluación; y se advierte de que cualquier modificación de la normativa en materia de responsabilidad de productos exigiría la actualización de la normativa en materia de seguridad de productos (Directiva 2001/95/CE).

\footnotetext{
${ }^{8}$ https://ec.europa.eu/transparency/regexpert/index.cfm?do=groupDetail.groupMeetingDoc\&docid $=3660$ 8 ${ }^{9}$ COM (2020)64 final. https://eur-lex.europa.eu/legalcontent/en/TXT/?qid $=1593079180383 \&$ uri=CELEX\%3A52020DC0064 10 COM (2020)65 final. https://ec.europa.eu/info/sites/info/files/commission-white-paper-artificialintelligence-feb2020 es.pdf

${ }^{11}$ En la traducción española de la Propuesta, errónea a mi juicio, se menciona a los operadores finales, pero en la versión inglesa se recoge el término "backend operator", que es en realidad el operador inicial, como no podía ser de otro modo, pues el operador final se asemeja más al usuario y es el operador inicial o remoto el que puede coincidir más frecuentemente con el productor.
} 
Revista CESCO, N.․ $37 / 2021$

doi.org/10.18239/RCDC_2021.38.2742
Pilar Álvarez Olalla

Propuesta de Reglamento en materia de responsabilidad civil por el uso de inteligencia artificial, del Parlamento Europeo, de 20 de octubre

de 2020

Las instituciones de la UE trabajan ya en la modificación de la Directiva 85/374/CEE). En el Informe de la Comisión al Parlamento Europeo, al Consejo y al Comité Económico y Social Europeo de 7 de mayo de $2018^{12}$, la Comisión evalúa si la actual Directiva aborda convenientemente los dispositivos autónomos y la ciberseguridad. Se llega a la conclusión de que la Directiva es todavía un instrumento adecuado para abordar la responsabilidad por productos, pero se hace necesario replantearse el significado de conceptos como producto, productor, defecto o daño, así como la regulación de la carga de la prueba.

El Reglamento propuesto, pues, tendría un ámbito de aplicación subjetivo distinto a la normativa en materia de productos defectuosos, pues quedaría limitado a regular la responsabilidad del "operador" ya sea el operador inicial o el final, justificándose esta responsabilidad en el hecho de que quien crea o mantiene un riesgo para el público; esto es, quien controla el riesgo asociado a un sistema de IA, es responsable si dicho riesgo se materializa en la causación de daños. La Propuesta, en su Preámbulo, equipara la responsabilidad del operador a la del propietario de un coche en la actualidad. Sin embargo, la cuestión de quién puede ser considerado "operador" se complica pues, cuanto más sofisticado y autónomo es el sistema y mayor es el impacto y la influencia del algoritmo en el funcionamiento del sistema, la persona encargada de controlar remotamente el dispositivo podría también considerarse "operador", en la medida en que también controla el riesgo. "Operador Final" o próximo sería, de este modo, la "persona física o jurídica" que ejerce un grado de control sobre un riesgo asociado a la operación y el funcionamiento de un sistema de IA, y que se beneficia de su funcionamiento. "Operador Inicial", por el contrario, sería la "persona física o jurídica" que define, de forma continuada, las características de la tecnología, proporciona datos y un servicio de apoyo final de base esencial y, por tanto, ejerce también un grado de control sobre el riesgo asociado a la operación y el funcionamiento del sistema (suministrando programas informáticos, suministrando datos de tráfico en un sistema de navegación, o suministrando programas de entrenamiento en un reloj inteligente, por ej.). Todo ello siendo "ejercicio del control" cualquier acción del operador que influya en el funcionamiento del sistema de IA. Ahora bien, si el "operador inicial" tiene la condición de productor conforme a la Directiva de productos defectuosos, la Propuesta prevé que a este sujeto le sea de aplicación preferente la Directiva. Además, se sugiere que el usuario puede ser considerado responsable si tiene la condición de "operador final". En caso de no tenerla, podría ser considerado solo responsable por un uso negligente o doloso frente a tercero, esto es, en virtud de un sistema de responsabilidad subjetiva.

Al margen de los responsables antes citados, en caso de daños causados por un tercero, serían de aplicación las normativas de los Estados miembros relativas a la responsabilidad subjetiva. Solo habrá que introducir correcciones en caso de que el tercero no sea rastreable o sea insolvente, previsiblemente a través de fondos de compensación.

\footnotetext{
${ }^{12}$ https://eur-lex.europa.eu/legal-content/ES/TXT/PDF/?uri=CELEX:52018DC0246\&from=FR
} 


\section{El sistema de responsabilidad civil diseñado en la propuesta}

La base del sistema de responsabilidad civil diseñado -que además es imperativo, en el sentido de que no cabe pacto en contrario entre operador y dañado, pues el mismo será nulo (art. 2.2 de la Propuesta) ${ }^{13}$ - se asienta sobre la diferencia entre Sistemas de Alto Riesgo, a los que se asigna un régimen de responsabilidad civil objetivo, y Sistemas que no son de Alto Riesgo, a los que se asigna un régimen de responsabilidad civil subjetivo. Quedan a salvo otros posibles regímenes de responsabilidad, como la responsabilidad contractual, cuando un vínculo de este tipo une a víctima con operador; o el régimen de productos defectuosos, de protección de consumidores, de lucha contra la discriminación, de protección laboral o del medioambiente; y ello tanto si se trata de un régimen específico aplicable al caso recogido en la legislación de la Unión como en las legislaciones nacionales. Veamos cómo se regula la cuestión en función de los distintos sistemas de inteligencia artificial previstos.

\subsection{Sistemas de Alto Riesgo. Responsabilidad objetiva}

Los Sistemas de Alto Riesgo son, tal y como los define el art. 3 de la Propuesta, aquellos "sistemas de inteligencia artificial ${ }^{14}$ que funcionan de forma autónoma ${ }^{15}$ con un potencial significativo para causar daños o perjuicios a una o más personas de manera aleatoria, quizás desconocidas e indeterminadas, y que exceden de lo que cabe esperar razonablemente. El potencial será significativo teniendo en cuenta la relación entre la gravedad del posible daño o perjuicio, el grado de autonomía en la toma de decisiones, la probabilidad de que el riesgo se materialice y el modo y contexto en que se utiliza el sistema de IA". Se tendrá en cuenta, pues, la potencialidad de causar daños al público en general, afectando, en su caso, a un número de personas desconocidas e indeterminadas; la gravedad del daño por su efecto lesivo en los derechos fundamentales, y el valor total de perjuicios. También se tendrá en cuenta el criterio de la evitabilidad de los daños. Se prevé que en el Anexo del Reglamento se enumeren todos los sistemas de IA de Alto Riesgo y los sectores críticos en los que se utilizan; si bien, la Comisión estará facultada para añadir, modificar o suprimir sistemas y sectores de la lista (art. 4.2).

Todo operador (inicial o final) de un Sistema calificado de Alto Riesgo, es responsable objetivamente de los daños causados por la actividad física o virtual de los dispositivos o procesos gobernados por dicho Sistema (art. 4.1). En virtud de ello, no podrá eludir su responsabilidad probando que empleó la diligencia debida, o que el daño lo causó el actuar autónomo del dispositivo. Solo quedará

${ }^{13}$ Y ello tanto si el acuerdo se ha concluido antes como después de causarse el daño.

${ }^{14}$ Según el propio art. 3 letra a) son "sistemas basados en programas informáticos o incorporados en dispositivos físicos que muestran un comportamiento que simula la inteligencia, entre otras cosas, mediante la recopilación y el tratamiento de datos, el análisis y la interpretación de su entorno y la actuación, con cierto grado de autonomía, ara lograr objetivos específicos".

${ }^{15}$ Es "autónomo" aquel sistema "que funciona interpretando determinados datos de entrada y utilizando un conjunto de instrucciones predeterminadas, sin limitarse a ellas, a pesar de que el comportamiento del sistema esté limitado y orientado a cumplir el objetivo que se le haya asignado y otras decisiones pertinentes de diseño tomadas por su desarrollador" (art. 3 letra b). 
exonerado al probar que el daño se causó por fuerza mayor. El problema, como ocurre en todos los supuestos de responsabilidad civil objetiva, es que será necesario delimitar, legal o jurisprudencialmente, entre lo que debe considerarse fuerza mayor, y el riesgo típico cubierto por la responsabilidad objetiva. No se hace referencia alguna al caso de que el daño sea causado por un tercero que pudiere interferir en el funcionamiento del sistema (a diferencia de lo que ocurre en el caso de la responsabilidad subjetiva de los sistemas no de Alto Riesgo, art. 8.3) por lo que el hecho de tercero no eliminará la responsabilidad objetiva del operador. Sin embargo, el art. 12.4 prevé una acción de regreso contra cualquier responsable civil del daño (el tercero) aunque, curiosamente, solo a favor de la aseguradora que haya indemnizado, y no a favor del propio operador. Sí está prevista la exoneración por culpa exclusiva de la víctima en el art. 10.1. Este sistema de responsabilidad objetiva prevalecerá sobre los regímenes nacionales de responsabilidad civil existentes (art. 4.5).

Respecto a los daños indemnizables causados por los Sistemas de Alto Riesgo son los siguientes:

- El daño corporal. Concretamente, el fallecimiento y los daños causados a la salud o a la integridad física, hasta un máximo de 2 millones de euros. Llama la atención en el Reglamento que lo indemnizable no es el daño corporal en sí sufrido por la víctima, sino más bien el daño emergente (costes médicos) o el lucro cesante (ingresos dejados de percibir) como consecuencia del daño corporal. Igualmente, en caso de muerte, la cuantía indemnizable se calcula teniendo en cuenta el importe de los costes del tratamiento médico que hubiere requerido la persona antes de su muerte, así como por el perjuicio económico sufrido antes del fallecimiento por el cese de su actividad, o por el incremento de las necesidades. También se reembolsarán los gastos funerarios, así como los gastos derivados del cumplimiento de los deberes de asistencia (también a concebidos no nacidos) que estuvieran a cargo del fallecido (pensión similar a la que hubiera tenido que pagar el fallecido, calculada conforme a la esperanza de vida del pensionista). Como se anticipaba, parece que el daño corporal sufrido por la víctima no es indemnizable, ni el daño moral de los familiares del fallecido, pues la indemnización se calcula en función de los costes o gastos, así como en función de los perjuicios económicos indirectos.

- Daños materiales y Daños morales que determinen daño económico calculado con la media de sus ingresos. Se considera daño moral el "considerable detrimento, objetivo y demostrable de los intereses personales". En el caso de los daños morales lo que se indemnizan son los gastos de tratamiento médico (daño económico emergente) y el perjuicio económico por el cese, suspensión temporal o reducción de su capacidad para generar ingresos, o el aumento de sus necesidades acreditado mediante certificado médico. De nuevo se comprueba que los daños indemnizables no abarcan el daño moral en sí, sino las consecuencias económicas del mismo. Se establece para los daños materiales y morales que causen perjuicio económico un límite conjunto 
de 1 millón de euros. El límite se aplicará cuando sean varios bienes materiales los afectados por un único funcionamiento de un sistema de IA de Alto Riesgo. En caso de que el damnificado tenga una acción de responsabilidad contractual contra el operador, no se abonará indemnización alguna en virtud de la responsabilidad extracontractual regulada en la Propuesta si el total de los daños materiales o morales a abonar no exceden de 500 euros. Se establece, pues, una franquicia de 500 euros cuando hay responsabilidad contractual concurrente.

Cuando sean varios los damnificados por un mismo funcionamiento de un sistema de IA de Alto Riesgo, la cuantía máxima ( 2 millones o 1 millón, en función del tipo de daño) no se sobrepasará, sino que se repartirá entre los perjudicados de forma proporcional al daño sufrido.

La cuantía, según se expresa en el apartado 16 del Preámbulo, es muy inferior a la establecida en la Directiva de Responsabilidad por Productos debido a que los límites que esta establece se aplican a los daños causados por una serie de productos, o una línea de productos, mientras que el Reglamento se refiere al daño causado a una persona por una única utilización de un sistema de IA.

Los operadores de Sistemas de Alto Riesgo tendrán obligación de concertar un seguro obligatorio (art. 4.4). La obligación se impone a ambos operadores. El seguro deberá cubrir los topes indemnizatorios previstos en la Propuesta. Sin embargo, el operador inicial o remoto puede optar entre cubrir la responsabilidad mediante un seguro de RC de productos o mediante un seguro empresarial. Se muestra en el Preámbulo la preocupación por el hecho de que, al no existir datos históricos sobre reclamaciones, sea difícil diseñar y encontrar en el mercado productos de seguro para las nuevas tecnologías.

La Propuesta establece un largo plazo de prescripción, de 30 años, para los daños causados por Sistemas de Alto Riesgo, cuyo "dies a quo" es la fecha en la que se produjo el daño (art. 7.1) cuando se trate de fallecimiento o daño a la salud o a la integridad física.

Para los daños materiales, o morales con perjuicio económico indemnizable, el plazo es de 10 años desde que se produjo el menoscabo a los bienes o el perjuicio económico comprobable como consecuencia del daño moral, o 30 años desde la fecha en que tuvo lugar la operación de IA que causó el daño material o moral. De ambos plazos, será preferido el plazo que venza antes (art. 7.2). Queda a salvo la legislación nacional en materia de suspensión o interrupción de plazos de prescripción (art. 7.3).

\subsection{Otros Sistemas de IA (no de Alto Riesgo). Responsabilidad Subjetiva}

Los sistemas de IA que no sean considerados de Alto Riesgo conforme al Anexo previsto en el futuro Reglamento, estarán sometidos a los regímenes nacionales en materia de responsabilidad por culpa. Sin embargo, se trata de una 
responsabilidad cuasiobjetiva, o con inversión de la carga de la prueba de la culpa ${ }^{16}$, pues el art. 8.1 establece que el operador (inicial, final o ambos) estará sujeto a responsabilidad subjetiva respecto de todo daño o perjuicio causado por la actividad física o virtual del sistema; pero en el apartado 2 de el precepto se matiza esta afirmación "salvo que pueda demostrar que no tuvo culpa en el daño o perjuicio causado" basándose en uno de los siguientes motivos:

- El sistema de IA se activó sin su conocimiento, habiendo tomado todas las medidas razonables para evitar dicha activación fuera del control del operador.

- El operador prueba que desplegó la diligencia exigible a través de la realización de unas concretas acciones:

- Llevó a cabo una selección de un sistema de IA adecuado y debidamente certificado, para las tareas y capacidades pertinentes.

- Llevó a cabo una correcta puesta en funcionamiento del sistema de IA, acreditando que supervisó real y periódicamente el sistema de IA durante su funcionamiento e informó al productor de posibles irregularidades.

- Llevó a cabo un control de actividades y mantenimiento de la fiabilidad operativa, con instalación de actualizaciones disponibles.

Para exonerarse no cabe, pues, probar el empleo de cualquier diligencia que pudiera ser exigible, sino tendrá que probar, bien que el sistema se activó sin su conocimiento, bien que cumplimentó todos y cada uno de los estándares de diligencia enunciados. Parece desprenderse del texto de la Propuesta que, en efecto, el operador no podrá probar ausencia de culpa fundada en otras alegaciones. Ahora bien, se prevé que el estándar de diligencia se gradúe en función de si el operador es un consumidor o un profesional. El operador tendrá indemnizar aunque el daño probadamente haya sido debido a la interferencia de un tercero, pero solo en caso de que el tercero esté ilocalizable o sea insolvente. En este caso, la responsabilidad que se establece es de garante; el operador es responsable por hecho de otro, de modo subsidiario.

Se prevé un deber de colaboración entre las partes implicadas a fin de facilitar, en la medida de lo posible, la mayor transparencia en relación con el funcionamiento del sistema de IA. El "productor" de un sistema de IA tiene la obligación de cooperar con los operadores y las víctimas, a petición de cualquiera de ellos, y facilitarles información como medio para determinar responsabilidades, si ello está justificado por la relevancia de la demanda (art. 8.4). Los productores tendrán la obligación de designar a un representante en materia de responsabilidad civil de IA para responder a las solicitudes de los operadores de modo similar a los delegados de protección de datos.

En estos casos de responsabilidad cuasiobjetiva por daños causados por sistemas no de Alto Riesgo, la Propuesta remite la determinación del plazo de prescripción

16 Presunción de culpa, es la expresión recogida en la Resolución (vid. apartado 20 de la Memoria explicativa de la Resolución). 
aplicable, así como el límite de las indemnizaciones y el alcance de los daños indemnizables, a lo establecido en la legislación de los distintos Estados miembros. Consecuentemente, en España, y siendo corporales los daños acaecidos, será de aplicación orientativa lo establecido en el Baremo de la Ley 8/2004 (con la reforma operada por Ley 35/2015).

\section{Disposiciones comunes aplicables a Sistemas de IA ya sean o no de Alto Riesgo}

\subsection{Culpa Concurrente de la víctima}

El art. 10 de la Propuesta prevé una reducción de la indemnización proporcional a la intervención de la víctima en el daño causado, en caso de culpa concurrente; y una exoneración cuando se trate de culpa exclusiva (que la víctima sea la única persona "a la que se le puede achacar el daño o perjuicio causado"). Tanto el operador como la persona afectada podrán utilizar los datos generados por el sistema para probar sus respectivas pretensiones, todo ello de conformidad con el Reglamento 2016/679.

\subsection{Responsabilidad solidaria de los operadores y acciones de regreso}

La Propuesta prevé la solidaridad de los operadores a la hora de hacer frente a la acción de reclamación de la víctima. Si solo hay un operador y el mismo es también productor, la aplicación del Reglamento prevalece sobre la Directiva de productos defectuosos (vid art. 11). Pero siendo varios los operadores, y uno de ellos el productor, las reglas aplicables son las siguientes: si el operador final es también el productor, la aplicación del Reglamento prevalecerá sobre la Directiva 85/347. $\mathrm{Si}$, por el contrario, la figura del productor coincide con la del operador inicial, tal y como se señaló anteriormente, respecto a él prevalecerá la Directiva. No aclara el Reglamento si la responsabilidad del operador inicial/productor elimina la responsabilidad del operador final, o si ambos responden solidariamente de la indemnización, aunque sea por títulos diferentes (uno, el operador inicial/productor por aplicación de la Directiva; y el otro, por aplicación del Reglamento). Parece que la primera interpretación es la preferible: cuando haya dos operadores, y el operador inicial sea a la vez el productor, este responderá en virtud de la aplicación de la Directiva de productos defectuosos, de ser esta aplicable, y ello impedirá dirigir la acción contra el operador final. De no ser aquella aplicable, por ejemplo, por no acreditarse el defecto del sistema, entendemos que debería aplicarse a ambos operadores la responsabilidad prevista en el Reglamento.

Respecto a las acciones de regreso, el art. 12 establece un requisito para el ejercicio de las mismas entre operadores: que el operador que la ejercita haya abonado a la víctima la totalidad de la indemnización a que esta tenga derecho conforme al Reglamento. Tanto si abonó la indemnización en virtud de la responsabilidad objetiva (sistemas de Alto Riesgo) o subjetiva (sistemas no de Alto Riesgo), la regla de reparto entre los distintos operadores en vía de regreso será la proporcionalidad a los respectivos niveles de control del riesgo. Se 
establece la solidaridad de los operadores también en vía de regreso, para cubrir al insolvente por su parte proporcional, frente al operador que abonó la totalidad de la indemnización.

Se establece también una acción de regreso del operador que abonó la totalidad de la indemnización, frente al productor, cuando el daño haya sido causado por un defecto sistema de IA defectuoso.

Asimismo, se prevé una acción de regreso, como antes se señaló, solo por parte de la aseguradora del operador, frente a un posible tercero responsable civil, hasta cubrir el importe abonado por la aseguradora.

No está de más recordar, para finalizar, que la Comisión Europea, por su parte, ha aprobado con fecha 21 de abril de 2021 una Propuesta de Regulación en materia de Inteligencia Artificial (Artificial Intelligence Act) si bien la misma se refiere fundamentalmente a aspectos relativos a los requisitos a cumplimentar por parte de productores y desarrolladores, para implementar determinados sistemas de IA, más que a aspectos de responsabilidad civil. 